Z. Epileptol. 2021 · 34:355-359

https://doi.org/10.1007/s10309-021-00442-z

Angenommen: 24. August 2021

Online publiziert: 28. September 2021

(c) Der/die Autor(en) 2021

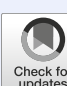

\section{Akut symptomatische Anfälle vs. beginnende Epilepsie nach Schlaganfall bei Erwachsenen und Kindern}

\author{
Indikationen zur Therapie bei Erwachsenen
}

\author{
Wolfgang Serles \\ Universitätsklinik für Neurologie, Medizinische Universität Wien, Wien, Österreich
}

\section{Zusammenfassung}

\section{In diesem Beitrag}

- Inzidenz des Schlaganfalls

- Inzidenz der Epilepsie

- Schlaganfall als Ursache von Epilepsie

- Akut symptomatische Anfälle (ASA) nach Schlaganfall

- Unprovozierte Anfälle und Epilepsie nach Schlaganfall

- ASA nach Schlaganfall - Inzidenz und Risikofaktoren

- Unprovozierter Anfall (= Epilepsie) nach Schlaganfall - Inzidenz und Risikofaktoren

- Indikationen zur Therapie Primärprophylaxe - ASA nach Schlaganfall - Primärprophylaxe - unprovozierter Anfall nach Schlaganfall • Sekundärprophylaxe - ASA nach Schlaganfall • Sekundärprophylaxe - unprovozierter Anfall nach Schlaganfall

- Welches Anfallsmedikament für die Sekundärprophylaxe nach Schlaganfall?

- ASA vs. Epilepsie im Kindesalter

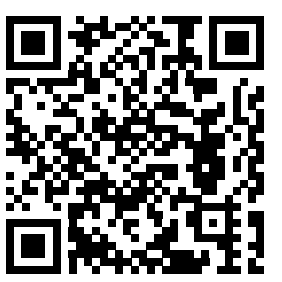

QR-Code scannen \& Beitrag online lesen
Mehr als ein Drittel der neu diagnostizierten Epilepsien bei Patienten über 60 Jahren haben einen abgelaufenen Schlaganfall als Ursache. Bei epileptischen Anfällen nach Schlaganfall müssen akut symptomatische Anfälle innerhalb 1 Woche nach Schlaganfall von unprovozierten epileptischen Anfällen ab 1 Woche nach Schlaganfall führen in $70 \%$ zu einem neuerlichen Anfall. Gemäß der Internationalen Liga gegen Epilepsie (ILAE) erfüllt letztere Konstellation bereits die Kriterien einer beginnenden Epilepsie, wofür nach früheren Definitionen zumindest 2 unprovozierte Anfälle notwendig waren. Akut symptomatische Anfälle stellen allerdings sowohl im Kindesalter als auch beim Erwachsenen einen Risikofaktor für die Entwicklung einer Epilepsie dar. Weitere Risikofaktoren sind die Größe des Infarktes und eine Lokalisation im Bereich der Hirnrinde. Die Studienlage zeigt weder ausreichende Evidenz für eine prophylaktische Gabe der Anfallsmedikation nach Schlaganfall noch für eine Therapie nach akut symptomatischem Anfall, obwohl dies häufig klinische Praxis ist. Daher sollte die medikamentöse Therapie nach der Akutphase des Schlaganfalls beendet werden. Bei Schlaganfallpatienten nach einem unprovozierten Anfall beim Erwachsenen wird eine lebenslange Therapie empfohlen. In der Regel stellt sich durch die Gabe eines Medikamentes ein zufriedenstellender Therapieerfolg ein. Bevorzugt werden sollten neuere Anfallsmedikamente, die besser verträglich sind und ein geringeres Interaktionspotenzial im Rahmen der Polypharmazie bei älteren Patienten haben.

Schlüsselwörter

Epilepsie · Schlaganfall · Epilepsie nach Schlaganfall · Akut symptomatischer Anfall · Therapie unterschieden werden. Erstere haben ein niedriges Rezidivrisiko, Zweitere hingegen
Der Schlaganfall nimmt ab dem 60. Lebensjahr exponentiell zu und ist die häufigste Ursache bleibender Behinderung. Die meisten Schlaganfälle sind Ischämien, gefolgt von intrazerebralen Blutungen. In den letzten Jahren gibt es ein zunehmendes Interesse an Epilepsien nach Schlaganfall, da diese eine Basis für ein Modell darstellen könnten, welcher pathophysiologische Prozess die Bereitschaft des Gehirns zu wiederholten epileptischen Anfällen determiniert (Epileptogenese). Daran knüpft sich die
Frage, ob durch Medikamente dieser Prozess verhindert werden kann. Unprovozierte epileptische Anfälle, die den Beginn einer Epilepsie darstellen, müssen dabei von akut symptomatischen Anfällen im Rahmen des akuten Schlaganfalls unterschieden werden. In den letzten Jahren wurden die Definitionen dafür vereinheitlicht und bessere epidemiologische Daten generiert. Die hier vorgestellten Therapieempfehlungen orientieren sich im Wesentlichen an den 
Richtlinien der Europäischen Schlaganfall Organisation (ESO).

\section{Inzidenz des Schlaganfalls}

In Österreich erleiden 24.000 Menschen jährlich einen Schlaganfall; $85 \%$ davon sind ischämische Infarkte, $10 \%$ primäre intrazerebrale Blutungen (IZB), 5\% Subarachnoidalblutungen $(S A B)$ und $0,5-1 \%$ zerebrale venöse Thrombosen. Die altersstandardisierte Inzidenz (nur Erstereignisse) in den deutschsprachigen Ländern beträgt ca. 150/100.000 Einwohner pro Jahr. Obwohl die Inzidenz, die Mortalität und die behinderungs- und krankheitskorrigierten Lebensjahre in den letzten 26 Jahren in Mitteleuropa abgenommen haben, bleiben aufgrund der zunehmenden Lebenserwartung und des exponentiellen Anstiegs des Schlaganfallrisikos im Alter die mit Behinderung nach Schlaganfall gelebten Lebensjahre hoch [7]. Entsprechend verhält sich auch das Risiko, nach einem Schlaganfall an einer Epilepsie zu erkranken.

Im Kindesalter ist der Schlaganfall selten, die Inzidenz beträgt 3,8/100.000 Kindern pro Jahr [12].

\section{Inzidenz der Epilepsie}

Die Epilepsieinzidenz beträgt 50/100.000 Einwohner pro Jahr und zeigt einen zweigipfeligen Verlauf mit Maximum im frühen Kindesalter und höheren Lebensalter [22].

\section{Schlaganfall als Ursache von Epilepsie}

Die Inzidenz von epileptischen Anfällen nach zerebrovaskulären Erkrankungen in der Rochester-Kohorte in Minnesota zwischen 1935 und 1984 betrug $11 \%$. In der Altersgruppe über 65 Jahre fanden sich in dieser Population in $55 \%$ zerebrovaskuläre Erkrankungen als Ursache für alle neu diagnostizierten Anfälle [1]. In einer neueren populationsbasierten Studie in Island, die akut symptomatische Anfälle ausschloss, zeigte sich eine zerebrovaskuläre Ursache in $9 \%$. In der Altersgruppe über 65 Jahre waren die häufigsten Ursachen degenerative Erkrankungen ( $25 \%)$, gefolgt von Schlaganfall mit $23 \%$ [22]. Im Rahmen der randomisierten Veteran Affairs Cooperati-
ve-Studie(VACS), in der über 60-Jährige mit Epilepsie eingeschlossen wurden, betrug der Anteil an Patienten mit ischämischen Schlaganfällen und Blutungen 36\%. Insbesondere im Rahmen der Altersepilepsie stellen epileptische Anfälle nach Schlaganfall in der Praxis einen relevanten Anteil dar [23].

Umgekehrt bestehen auch Hinweise für ein erhöhtes Risiko bei über 60-Jährigen Patienten mit neu diagnostizierter Epilepsie, einen späteren ersten Schlaganfall zu erleiden, was mit dem Vorliegen von Mikroangiopathien oder stummen Infarkten erklärt werden kann [6].

\section{Akut symptomatische Anfälle (ASA) nach Schlaganfall}

ASA sind epileptische Anfälle, die in enger zeitlicher Beziehung zu systemischen Veränderungen (z. B. toxisch oder metabolisch) oder einer direkten ZNS-Schädigung auftreten. Für den Schlaganfall definierte man diese als „Frühanfälle“ innerhalb von 7 Tagen, wobei sich ca. 50\% innerhalb der ersten $24 \mathrm{~h}$ manifestieren [2]. Pathophysiologisch werden neben dem Verlust des lonengleichgewichts an der Zellmembran eine erhöhte Glutamat-induzierte Exzitotoxizität sowie Perinfarktdepolarisationen angenommen [5].

Obwohl die Wahl des Zeitintervalls von 1 Woche pathophysiologisch arbiträr erscheint, beruht diese Definition v. a. auf der populationsbasierten Studie von Hesdorffer et al. 2009 bei Patienten nach SchädelHirn-Trauma, Schlaganfall und ZNS-Infektionen. In der Schlaganfallgruppe betrug das 10-Jahres-Risiko, nach einem ASA innerhalb der ersten Woche einen ersten unprovozierten Anfall (nach 1 Woche) zu erleiden, $30 \%$. Im Gegensatz dazu war das Rezidivrisiko nach einem ersten unprovozierten Anfall mit 70\% deutlich höher (entspricht dem Risiko nach 2 unprovozierten Anfällen) [18].

\section{Unprovozierte Anfälle und Epilepsie nach Schlaganfall}

Ein unprovozierter Anfall ist definiert als epileptischer Anfall ohne engen zeitlichen Bezug zu einer systemischen Veränderung oder einer akuten ZNS-Schädigung. Für den Schlaganfall definierte man dies als
„Spätanfälle" > 7 Tage nach dem Ereignis. Gemäß der neuen ILAE-Definition von Epilepsie mit der Wahrscheinlichkeit eines Rezidivrisikos innerhalb von 10 Jahren von zumindest $60 \%$ (adäquat dem Risiko nach 2 unprovozierten Anfällen) erfüllt damit der Schlaganfallpatient bereits die Kriterien einer Epilepsie (s. oben) (ס Abb. 1; $[9,11])$. Pathophysiologisch soll dafür eine Gliose mit meningozerebraler Narbenbildung verantwortlich sein.

\section{ASA nach Schlaganfall - Inzidenz und Risikofaktoren}

Die Inzidenz von ASA nach jeglichem Schlaganfall beträgt 3-6\%, dabei liegt die Inzidenz für arterielle Blutungen (IZB und $S A B)$ mit $10-16 \%$ höher und kann bei zerebralen venösen Infarkten mit kortikaler Beteiligung bis zu $40 \%$ ausmachen [10]. Die Risikofaktoren für einen ASA sind eine kortikale Lokalisation, eine Blutung (oder hämorrhagische Transformation eines ischämischen Infarktes), aber auch die Größe und die Schwere des Schlaganfalls sowie eine Lokalisation im vorderen Stromkreislauf (Versorgungsgebiet der A. carotis interna) [19]. Damit lässt sich auch innerhalb der Einteilung des ischämischen Schlaganfalls nach klinischen Symptomen (sog. Bamford- oder OCSP-Klassifikation) eine höhere Inzidenz bei großen Infarkten der vorderen Strombahn („total anterior circulation infarct") im Vergleich zu partiellen oder subkortikalen, lakunären Infarkten erklären [4]. Neuere Daten zeigen eine erhöhte Inzidenz für ASA nach Schlaganfall bei Patienten mit nichtneurologischen akuten Infektionen und einem niedrigeren funktionellen Status vor dem Schlaganfall [28].

\section{Unprovozierter Anfall (= Epilepsie) nach Schlaganfall - Inzidenz und Risikofaktoren}

Die Inzidenz für einen unprovozierten Anfall mehr als 7 Tage nach einem zerebrovaskulären Ereignis beträgt 10-12\% innerhalb eines Zeitraumes von 5 bis 10 Jahren. Dabei ist die Inzidenz gleich bezüglich der Art des Schlaganfalls (Blutung oder Ischämie) [19]. Bekannte Risikofaktoren für einen unprovozierten Anfall nach Schlaganfall sind kortikale Lokalisation, die Grö- 


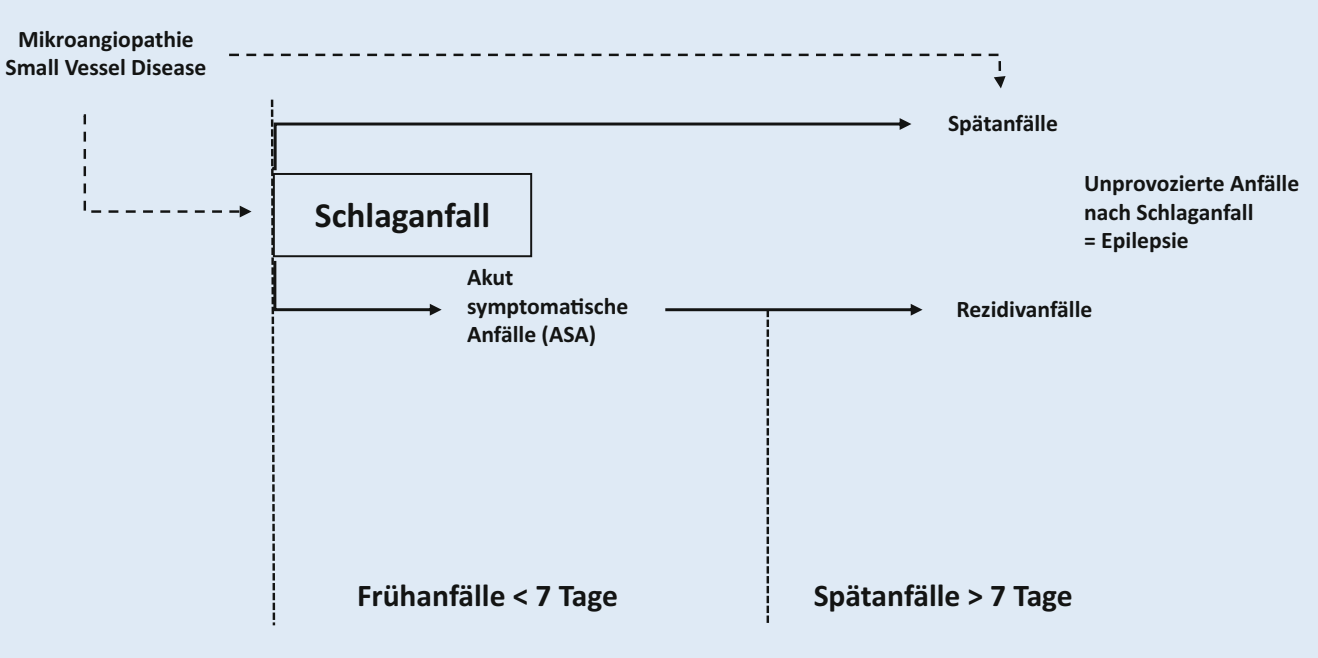

Abb. $1 \varangle$ Klassifikation von epileptischen Anfällen nach Schlaganfall. (Mod. nach Derex et al. [9]) ße der Läsion (z.B. > $10 \mathrm{ml}$ Volumen bei intrazerebralen Blutungen) und akut symptomatische Anfälle innerhalb von 7 Tagen [17]. Huttunen et al. konnten zeigen, dass nach SAB außer ASA auch die Schwere der Blutung (Hunt \&Hess Grade III-V) sowie parenchymale Hämatome von $>15 \mathrm{~cm}^{3}$ unabhängige Risikofaktoren für die Entwicklung einer Epilepsie darstellen [20].

\section{Indikationen zur Therapie}

\section{Primärprophylaxe - ASA nach Schlaganfall}

Es gibt keine ausreichende Evidenz, dass die Gabe eines Anfallsmedikaments vor einem etwaigen ASA diesen verhindert. Das generelle Risiko für einen ASA ist mit 3-6\% niedrig, und auch individuelle Risiken, z. B. nach Blutungen und kortikaler Beteiligung, übersteigen $40 \%$ nicht [19].

Ebenso ist ungeklärt, ob das funktionelle Outcome oder die Mortalität des Schlaganfalls durch einen singulären Anfall beeinflusst wird.

Sollte eine Medikation begonnen worden sein, so ist darauf zu achten, diese nach der Akutphase zu beenden. Allerdings ist diese nicht ausreichend definiert, und die Angaben schwanken zwischen 4 Wochen und 6 Monaten.

\section{Primärprophylaxe - unprovozierter Anfall nach Schlaganfall}

Es gibt keine Evidenz, dass eine medikamentöse Therapie einen unprovozierten
Anfall nach Schlaganfall verhindert. Das generelle Risiko für einen unprovozierten Anfall nach Schlaganfall innerhalb von 5 bis 10 Jahren beträgt nur 10-12\% und nimmt erst mit der Dauer des Intervalls zu. In Fällen mit erhöhtem Risiko nach Blutungen oder malignem Mediainfarkt nach dekompressiver Kraniektomie liegt das Risiko in der Regel um 50\% [17, 25].

Galovic et al. konnten anhand eines prädiktiven Scores mit 5 Parametern (,,severity of stroke", "large-artery atherosclerotic aetiology", "early seizures", "cortical involvement" und "territory of middle cerebral artery involvement" - SeLECT) in der Gruppe mit der höchsten Punkteanzahl ein Risiko von bis zu $63 \%$ für einen unprovozierten Anfall innerhalb eines Jahres vorhersagen [14]. Weitere Studien müssen zeigen, ob diese Patienten von einer medikamentösen Therapie profitieren. Sollte eine Medikation begonnen worden sein, so ist darauf zu achten, diese nach der Akutphase zu beenden.

\section{Sekundärprophylaxe - ASA nach Schlaganfall}

Eine Anfallsmedikationsgabe nach ASA mit dem Ziel, einen weiteren Anfall zu verhindern, wird nicht empfohlen. Das Rezidivrisiko für einen neuerlichen Anfall ist mit 10-20\% niedrig. Das 10-Jahres-Risiko für einen unprovozierten Anfall beträgt $30 \%$. In der klinischen Praxis wird eine Sekundärprophylaxe aber häufig dennoch durchgeführt, sollte aber nach der Akutphase wieder beendet werden [19].
Sekundärprophylaxe - unprovozierter Anfall nach Schlaganfall

Über etwaige Vorteile einer Anfallsmedikationsgabe nach einem unprovozierten Anfall nach Schlaganfall im Vergleich zu keiner Gabe existieren keine randomisierten Studien. Aufgrund der Höhe des Rezidivrisikos von zumindest $60 \%$ innerhalb von 10 Jahren wird auf Basis der Epilepsiedefinition der ILAE aber eine Therapie empfohlen [19].

Die Therapie sollte lebenslang fortgesetzt werden, da das Rezidivrisiko bei läsionellen Epilepsien nach Absetzen über $50 \%$ beträgt [21].

Die Entscheidung über einen Absetzversuch kann nur im Einzelfall gemeinsam mit dem Patienten getroffen werden.

\section{Welches Anfallsmedikament für die Sekundärprophylaxe nach Schlaganfall?}

Für die Wahl der Anfallsmedikation in der Folge eines unprovozierten Anfalls nach Schlaganfall gibt es keine klaren Empfehlungen. In 2 randomisierten Studien mit sehr kleinen Fallzahlen, die eine Lamotrigin-bzw. Levetiracetam-Monotherapie mit Carbamazepin bei Patienten mit ischämischen Infarkten bzw. Ischämien und IZB verglichen, zeigten sich keine Unterschiede in der Wirksamkeit bei besserer Verträglichkeit der neueren Anfallsmedikamente $[8,16]$.

In der randomisierten VACS-Studie von Rowan et al. wurden 593 Patienten über 
60 Jahre (570 Männer, 52\% mit Schlaganfall) mit De-novo-Anfällen in 3 Gruppen mit Lamotrigin, Gabapentin oder Carbamazepin behandelt. Der Anteil an anfallsfreien Patienten nach 1 Jahr war gleich, die Verträglichkeit von Lamotrigin und Gabapentin jedoch signifikant besser als in der Carbamazepin-Gruppe [24].

Aufgrund der Kritik an der Verwendung der nicht retardierten Carbamazepin-Tablette (und damit schlechteren Verträglichkeit) konnte in einem ähnlichen Vergleich bei 359 Patienten (über 60 Jahre, $66 \%$ mitzerebrovaskulären Erkrankungen) dieses Ergebnis für Levetiracetam, allerdings nicht signifikant für Lamotrigin, im Vergleich zur retardierten CarbamazepinForm reproduziert werden [27].

Die Epilepsie nach Schlaganfall ist prinzipiell gut zu behandeln. In einer retrospektiven Studie von 1148 Patienten mit struktureller Epilepsie im Alter von mehr als 16 Jahren in einem tertiären Epilepsiezentrum zeigte sich die zugrunde liegende Ätiologie als prognostischer Faktor bezüglich der medikamentösen Therapieresistenz. Im Vergleich zu Patienten mit Hippocampussklerose und assoziierter kortikaler Läsion im Temporallappen (duale Pathologie) mit einer medikamentösen Therapieresistenz in $97 \%$ fand sich diese bei Epilepsie nach Schlaganfall in nur $46 \%$ [26]. In einer weiteren retrospektiven Studie wurde eine medikamentöse Therapieresistenz von unter $5 \%$ erhoben [15].

\section{ASA vs. Epilepsie im Kindesalter}

Die Inzidenz von ASA nach Schlaganfall ist bei Kindern wesentlich häufiger als im Erwachsenenalter und beträgt 20-30\%. Wenn man neonatale ASA (innerhalb der ersten 28 Lebenstage) hinzurechnet, liegen die Zahlen noch höher. Umso jünger die Patienten zum Zeitpunkt des Insultes sind und umso häufiger und länger die ASA dauern, desto größer scheint das Epilepsierisiko zu sein [13]. In einer retrospektiven populationsbasierten Studie in Kalifornien bei Kindern im Alter zwischen 29 Tagen und 19 Jahren (neonatale Anfälle waren ausgenommen) belief sich das kumulative Risiko für einen ersten unprovozierten Anfall (definiert als > 30 Tage nach dem Schlaganfall) auf $16 \%$ innerhalb von 5 Jah- ren und $33 \%$ innerhalb von 10 Jahren. Kinder mit akuten Anfällen zum Zeitpunkt des Schlaganfalls zeigten ein mehr als 4-fach erhöhtes Risiko für eine aktive Epilepsie, definiert als zumindest 1 unprovozierter Anfall unter Medikation oder weniger als 6 Monate anfallsfrei ohne Medikation [12].

Eine rezente retrospektive monozentrische Studie zeigte bei Kindern älter als 28 Tage mit ASA innerhalb von 7 Tagen aufgrund eines akuten Infarktes eine kumulative Epilepsieinzidenz von lediglich $7 \%$ innerhalb von 2 Jahren. Etwas höhere Inzidenzen fanden sich bei innerhalb der ersten 28 Tage symptomatischen Kindern (11\%) und Kindern, die erst nach 28 Tagen aufgrund eines perinatalen Infarktes, der nicht bemerkt wurde, symptomatisch wurden (19\%). ASA stellten einen Risikofaktor für die Entwicklung einer Epilepsie dar [3]. Therapierichtlinien im Kindesalter existieren nicht.

\section{Fazit für die Praxis}

- Schlaganfall ist eine häufige Ursache für neu diagnostizierte Epilepsien bei über 60-Jährigen (30-40\%).

- Akut symptomatische Anfälle innerhalb von 7 Tagen sind im Kindesalter häufig, beim Erwachsenen seltener (3-6\%, Inzidenz bei Blutungen höher).

- Akut symptomatische Anfälle haben beim Erwachsenen ein niedriges Rezidivrisiko (30\%).

- Unprovozierte Anfälle mehr als 7 Tage nach Schlaganfall haben ein hohes Rezidivrisiko (70\%) und erfüllen die Kriterien für eine Epilepsie. Die Inzidenz beträgt 10-12\%. Die Epilepsieinzidenz im Kindesalter (die Neonatalperiode ausgenommen) dürfte ähnlich sein.

- Die Risikofaktoren für eine Epilepsie nach Schlaganfall sind kortikale Lokalisation, die Größe der Läsion und ASA.

- Es gibt keine Evidenz für eine Primärprophylaxe zur Verhinderung von ASA oder ersten unprovozierten Anfällen.

- Es gibt keine Evidenz für eine Sekundärprophylaxe nach akut symptomatischem Anfall.

- Wichtig ist das Absetzen der Anfallsmedikation nach der Akutphase nach ASA bzw. etwaiger prophylaktischer Gabe.

- Es besteht eine Indikation für eine medikamentöse Therapie bei einem unprovozierten Anfall nach Schlaganfall.

- Die medikamentöse Therapieresistenz bei Epilepsie nach Schlaganfall ist gering.

- Es wird eine Monotherapie empfohlen, wobei neuere Anfallsmedikamente eine bessere Verträglichkeit aufweisen.

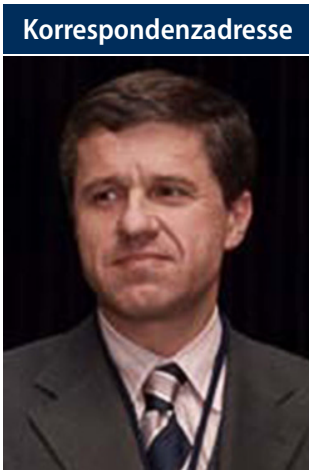

Prof. Dr. Wolfgang Serles

Universitätsklinik für Neurologie, Medizinische Universität Wien

Währinger Gürtel 18-20, 1090 Wien, Österreich wolfgang.serles@meduniwien.ac.at

Funding. Open access funding provided by Medical University of Vienna.

\section{Einhaltung ethischer Richtlinien}

Interessenkonflikt. W. Serles gibt an, dass kein Interessenkonflikt besteht.

Für diesen Beitrag wurden vom Autor keine Studien an Menschen oder Tieren durchgeführt. Für die aufgeführten Studien gelten die jeweils dort angegebenen ethischen Richtlinien.

Open Access. Dieser Artikel wird unter der Creative Commons Namensnennung 4.0 International Lizenz veröffentlicht, welche die Nutzung, Vervielfältigung Bearbeitung, Verbreitung und Wiedergabe in jeglichem Medium und Format erlaubt, sofern Sie den/die ursprünglichen Autor(en) und die Quelle ordnungsgemäß nennen, einen Link zur Creative Commons Lizenz beifügen und angeben, ob Änderungen vorgenommen wurden.

Die in diesem Artikel enthaltenen Bilder und sonstiges Drittmaterial unterliegen ebenfalls der genannten Creative Commons Lizenz, sofern sich aus der Abbildungslegende nichts anderes ergibt. Sofern das betreffende Material nicht unter der genannten Creative Commons Lizenz steht und die betreffende Handlung nicht nach gesetzlichen Vorschriften erlaubt ist, ist für die oben aufgeführten Weiterverwendungen des Materials die Einwilligung des jeweiligen Rechteinhabers einzuholen.

Weitere Details zur Lizenz entnehmen Sie bitte de Lizenzinformation auf http://creativecommons.org/ licenses/by/4.0/deed.de.

\section{Literatur}

1. Annegers JF, Rocca WA, Hauser WA (1996) Causes of epilepsy: contributions of the Rochester epidemiology project. Mayo Clin Proc 71:570-575

2. Beghi E, Carpio A, Forsgren L, Hesdorffer DC, Malmgren K, Sander JW, Tomson T, Hauser WA 
(2010) Recommendation for a definition of acute symptomatic seizure. Epilepsia 51:671-675

3. Billinghurst LL, Beslow LA, Abend NS, Uohara M, Jastrzab L, Licht DJ, Ichord RN (2017) Incidence and predictors of epilepsy after pediatric arterial ischemic stroke. Neurology 88:630-637

4. Burn J, Dennis M, Bamford J, SandercockP, Wade D, Warlow C (1997) Epileptic seizures after a first stroke: the Oxfordshire Community Stroke Project. BMJ315:1582-1587

5. Camilo O, Goldstein LB (2004) Seizures andepilepsy after ischemic stroke. Stroke 35:1769-1775

6. Cleary P, Shorvon S, Tallis R (2004) Late-onset seizures as a predictor of subsequent stroke. Lancet 363:1184-1186

7. GBD 2016 Stroke Collaborators (2019) Global, regional, and national burden of stroke, 1990-2016: a systematic analysis for the Global Burden of Disease Study 2016. Lancet Neurol 18:439-458

8. Consoli D, Bosco D, Postorino P, Galati F, Plastino M, Perticoni GF, Ottonello GA, Passarella B, Ricci S, Neri G, Toni D, Study E (2012) Levetiracetam versus carbamazepine in patients with late poststroke seizures: a multicenter prospective randomized open-label study (EpIC Project). Cerebrovasc Dis 34:282-289

9. Derex L, Rheims S, Peter-Derex L (2021) Seizures and epilepsy after intracerebral hemorrhage: an update. J Neurol 268:2605-2615

10. Ferro JM, Canhao P, Bousser MG, Stam J, Barinagarrementeria $F$, Investigators I (2008) Early seizures in cerebral vein and dural sinus thrombosis: risk factors and role of antiepileptics. Stroke 39:1152-1158

11. Fisher RS, Acevedo C, Arzimanoglou A, Bogacz A, Cross JH, Elger CE, Engel J Jr, Forsgren L, French JA, Glynn M, Hesdorffer DC, Lee BI, Mathern GW, Moshe SL, Perucca E, Scheffer IE, Tomson T, Watanabe M, Wiebe S (2014) ILAE official report: a practical clinical definition of epilepsy. Epilepsia 55:475-482

12. Fox CK, Glass HC, Sidney S, Lowenstein DH, Fullerton $\mathrm{HJ}$ (2013) Acute seizures predict epilepsy after childhood stroke. Ann Neurol 74:249-256

13. Fox CK, Mackay MT, Dowling MM, Pergami $P$, Titomanlio L, Deveber G, Investigators S (2017) Prolonged or recurrent acute seizures after pediatric arterial ischemic stroke are associated with increasing epilepsy risk. Dev Med Child Neurol 59:38-44

14. Galovic M, Dohler N, Erdelyi-Canavese B, Felbecker A, Siebel P, Conrad J, Evers S, Winklehner M, von Oertzen TJ, Haring HP, Serafini A, Gregoraci G, Valente M, Janes F, Gigli GL, Keezer MR, Duncan JS, Sander JW, Koepp MJ, Tettenborn B (2018) Prediction of late seizures after ischaemic stroke with a novel prognostic model (the SeLECT score): a multivariable prediction model development and validation study. Lancet Neurol 17:143-152

15. Gasparini S, Ferlazzo E, Beghi E, Sofia V, Mumoli L, Labate A, Cianci V, Fatuzzo D, Bellavia MA, Arcudi L, Russo E, De Sarro G, Gambardella A, Aguglia U (2015) Epilepsy associated with Leukoaraiosis mainly affects temporal lobe: a casual or causal relationship? Epilepsy Res 109:1-8

16. Gilad R, Sadeh M, Rapoport A, Dabby R, Boaz M, LamplY (2007) Monotherapy of lamotrigine versus carbamazepine in patients with poststroke seizure. Clin Neuropharmacol 30:189-195

17. Haapaniemi E, Strbian D, Rossi C, Putaala J, Sipi T, Mustanoja S, Sairanen T, Curtze S, Satopaa J, Roivainen R, Kaste M, Cordonnier C, Tatlisumak T, Meretoja A (2014) The CAVE score for predicting

\section{Acute symptomatic seizures vs. onset of epilepsy after stroke in adults and children. Indications for treatment in adults}

More than one third of newly diagnosed epilepsies in patients over 60 years old are caused by a previous stroke. Epileptic seizures after stroke are defined as acute symptomatic seizures occurring within 7 days or unprovoked epileptic seizures more than 7 days after stroke. Acute symptomatic seizures bear a low risk of recurrence as opposed to unprovoked seizures, which recur in $70 \%$. Therefore, the International League Against Epilepsy (ILAE) has defined a single unprovoked seizure more than 7 days after stroke as epilepsy, contrary to previous requirements of at least two unprovoked seizures; however, acute symptomatic seizures are a risk factor for the development of epilepsy in children and adults. Further risk factors are the size of the infarct and cortical involvement. There is no clear evidence for prophylactic antiseizure treatment after stroke or after acute symptomatic seizures, although in real life medication is often initiated. In these cases, medication should be stopped after the acute phase of the insult. There is weak but sufficient evidence to start antiseizure medication after one unprovoked seizure, i.e. post-stroke epilepsy. Life-long treatment is recommended in adults. Usually, post-stroke epilepsy can be well controlled.

Preferred antiseizure drugs are newer compounds, which are better tolerated and have less potential to interact with co-medications in older patients.

\section{Keywords}

Epilepsy $\cdot$ Stroke $\cdot$ Post-stroke epilepsy $\cdot$ Acute symptomatic seizure $\cdot$ Treatment

late seizures after intracerebral hemorrhage. Stroke 45:1971-1976

18. Hesdorffer DC, Benn EK, Cascino GD, Hauser WA (2009) Is a first acute symptomatic seizure epilepsy? Mortality and risk for recurrent seizure. Epilepsia 50:1102-1108

19. Holtkamp M, Beghi E, Benninger F, Kalviainen R, Rocamora R, Christensen H (2017) European Stroke Organisation guidelines for the management of post-stroke seizures and epilepsy. Eur Stroke J 2:103-115

20. Huttunen J, Kurki MI, von und zu Fraunberg $M$, Koivisto T, Ronkainen A, Rinne J, Jaaskelainen JE, Kalviainen R, Immonen A (2015) Epilepsy after aneurysmal subarachnoid hemorrhage: a population-based, long-term follow-up study. Neurology 84:2229-2237

21. Lossius MI, Hessen E, Mowinckel P, Stavem K, Erikssen J, Gulbrandsen P, Gjerstad L (2008) Consequences of antiepileptic drug withdrawal: a randomized, double-blind study (Akershus Study). Epilepsia 49:455-463

22. Olafsson $E$, Ludvigsson $P$, Gudmundsson $G$, Hesdorffer D, Kjartansson O, Hauser WA (2005) Incidence of unprovoked seizures and epilepsy in Iceland and assessment of the epilepsy syndrome classification: a prospective study. Lancet Neurol 4:627-634

23. Ramsay RE, Rowan AJ, Pryor FM (2004) Special considerations in treating the elderly patient with epilepsy. Neurology 62:S24-S29

24. Rowan AJ, Ramsay RE, Collins JF, Pryor F, Boardman KD, Uthman BM, Spitz M, Frederick T, Towne A, Carter GS, Marks W, Felicetta J, Tomyanovich ML, VA Cooperative Study 428 Group (2005) New onset geriatric epilepsy: a randomized study of gabapentin, lamotrigine, and carbamazepine. Neurology 64:1868-1873

25. Santamarina E, Sueiras M, Toledo M, Guzman L, Torne R, Riveiro M, Quintana M, Salas Puig X, Sahuquillo J, Alvarez Sabin J (2015) Epilepsy in patients with malignant middle cerebral artery infarcts and decompressive craniectomies. Epilepsy Res 112:130-136

26. Semah F, Picot MC, Adam C, Broglin D, Arzimanoglou A, Bazin B, Cavalcanti D, Baulac M (1998) Is the underlying cause of epilepsy a major prognostic factor for recurrence? Neurology 51:1256-1262

27. Werhahn KJ, TrinkaE, Dobesberger J, Unterberger I, Baum P, Deckert-Schmitz M, Kniess T, Schmitz B, Bernedo V, Ruckes C, Ehrlich A, Kramer G (2015) $A$ randomized, double-blind comparison of antiepileptic drug treatment in the elderly with new-onset focal epilepsy. Epilepsia 56:450-459

28. Zollner JP, Misselwitz B, Kaps M, Stein M, Konczalla J, Roth C, Krakow K, Steinmetz H, Rosenow F, Strzelczyk A (2020) National Institutes of Health Stroke Scale (NIHSS) on admission predicts acute symptomatic seizure risk in ischemic stroke: a population-based study involving 135,117 cases. Sci Rep 10:3779 